\title{
The metabolic syndrome in pregnancy and its association with child telomere length
}

\author{
Dale McAninch $^{1,2} \cdot$ Tina Bianco-Miotto ${ }^{1,3}$ (D) Kathy L. Gatford ${ }^{1,2}$ (D) Shalem Y. Leemaqz ${ }^{1,2,4}$. \\ Prabha H. Andraweera ${ }^{1,2}$. Amy Garrett ${ }^{1,2}$. Michelle D. Plummer ${ }^{1,2}$ - Gus A. Dekker ${ }^{1,5}$ - Claire T. Roberts ${ }^{1,2,4}$ (D) \\ Lisa G. Smithers ${ }^{1,6}$ (D) Jessica A. Grieger ${ }^{1,2}$ (D)
}

Received: 9 March 2020 / Accepted: 24 June 2020 / Published online: 29 July 2020

(C) Springer-Verlag GmbH Germany, part of Springer Nature 2020

\begin{abstract}
Aims/hypothesis The aim of this study was to determine whether presence of the metabolic syndrome in pregnancy associates with child telomere length or child anthropometry (weight, BMI) and BP, measured at 10 years of age.

Methods The Screening for Pregnancy Endpoints study (SCOPE) was a multicentre, international prospective cohort of nulliparous pregnant women recruited from Australia, New Zealand, Ireland and the UK $(N=5628)$. The current analysis is a 10 year follow-up of SCOPE pregnant women and their children, from the Australian cohort. Clinical data collected at 14-16 weeks' gestation during the SCOPE study were used to diagnose the metabolic syndrome using IDF criteria. Telomere length, a biomarker of ageing, was assessed by quantitative PCR from children's saliva collected at 10 years of age.

Results In women who completed follow-up $(n=255), 20 \%$ had the metabolic syndrome in pregnancy. After adjusting for a range of confounders, children of mothers who had the metabolic syndrome in pregnancy had $14 \%$ shorter telomeres than children of mothers without the metabolic syndrome in pregnancy (mean difference -0.36 [95\% CI $-0.74,0.01]$ ). Height- and weight-for-age, and BMI $z$ scores were similar in children of mothers who did and did not have the metabolic syndrome during pregnancy.

Conclusions/interpretation Children of mothers who had the metabolic syndrome in pregnancy have shorter telomeres, a biomarker of accelerated ageing. These findings warrant further studies in larger cohorts of children, as well as investigations into whether telomere length measured in cord blood associates with telomere length in childhood.
\end{abstract}

Keywords Cardiovascular · Children · Developmental programming · Maternal $\cdot$ Metabolic syndrome $\cdot$ Obesity $\cdot$ Offspring · Pregnancy $\cdot$ Telomere length

Electronic supplementary material The online version of this article (https://doi.org/10.1007/s00125-020-05242-0) contains peer-reviewed but unedited supplementary material, which is available to authorised users.

Jessica A. Grieger

jessica.grieger@adelaide.edu.au

1 Robinson Research Institute, University of Adelaide, North Adelaide, SA 5005, Australia

2 Adelaide Medical School, University of Adelaide, Adelaide, Australia

3 Waite Research Institute, School of Agriculture, Food and Wine, University of Adelaide, Adelaide, SA, Australia

4 College of Medicine and Public Health, Flinders University, Bedford Park, SA, Australia

5 Women and Children's Division, Lyell McEwin Hospital, University of Adelaide, Adelaide, SA, Australia

6 School of Public Health, University of Adelaide, Adelaide, SA, Australia

\author{
Abbreviations \\ BMIz BMI $z$ scores \\ GDM Gestational diabetes \\ HFAz Height-for-age $z$ scores \\ qPCR Quantitative PCR \\ SCOPE Screening for Pregnancy Endpoints study \\ SEI Socioeconomic index \\ WFAz Weight-for-age $z$ scores
}

\section{Introduction}

Telomeres are repeating DNA sequences located at the ends of chromosomes that progressively shorten with each round of cell division [1]. Their main function is to protect the chromosomes from DNA damage thus helping to maintain genomic stability. Cells are generally unaffected by a small amount of 


\section{Research in context}

\section{What is already known about this subject?}

- The metabolic syndrome is a clustering of cardiovascular risk factors, which in adults associates with an increased risk for cardiovascular diseases

- Telomere length is a biomarker of ageing and is associated with the development of future chronic diseases

What is the key question?

- Do children of mothers who had the metabolic syndrome in pregnancy have shorter telomeres than children of mothers who did not have the metabolic syndrome?

What are the new findings?

- Children of mothers who had the metabolic syndrome in pregnancy had $14 \%$ shorter telomeres than children of mothers who did not have the metabolic syndrome

- Height- and weight-for-age and BMI z scores were similar in children of mothers who did and did not have the metabolic syndrome in pregnancy

How might this impact on clinical practice in the foreseeable future?

- Early assessment of telomere length in children may provide insight into their potential future chronic disease risk

telomere erosion; however, once they reach a critically short length, chromosome ends become uncapped causing cell senescence, signalling the arrest of cell proliferation and subsequently leading to apoptosis [2]. Oxidative stress and inflammation shorten telomeres in vitro [3]. Consistent with this, there is human cross-sectional evidence associating shorter telomeres with oxidative stress and inflammation [4]. Shorter telomeres are a marker of the cumulative damage to which the cell has been exposed [5] and, in adults, is associated with diabetes [6], cancer [7], cardiovascular disease [8] and all-cause mortality [9].

Antenatal factors are a particularly important determinant of telomere length [10]. Adverse exposures in pregnancy, such as maternal stress, smoking, exposure to second-hand smoke and higher levels of air pollution, associate with shorter telomeres measured in cord blood [11-13], placenta [14] and fetal lung tissue [15]. For every $1 \mathrm{~kg} / \mathrm{m}^{2}$ increase in prepregnancy BMI, telomeres measured in cord blood were $0.50 \%$ shorter, while telomeres measured in the placenta were $0.66 \%$ shorter [16]. Gestational diabetes (GDM) in pregnancy was associated with shorter telomeres measured in cord blood in one study [17] but not in others [18, 19]. Importantly, exposure to maternal obesity or GDM is associated with a heightened risk for future cardiovascular disease in both the mother and the child $[20,21]$. Furthermore, even before frank cardiovascular or metabolic diseases develop, children of complicated pregnancies already have markers of elevated risk for these conditions, including higher BP [22], overweight or obesity [23], elevated lipid levels [24] and type 2 diabetes [25], compared with children born after an uncomplicated pregnancy.
Despite this evidence for associations between adverse pregnancy exposures and progeny telomere length at birth, it is not known whether these associations persist into childhood. The metabolic syndrome is a clustering of modifiable cardiovascular risk factors including abdominal obesity, hyperlipidaemia, raised BP and insulin resistance [26]. We have recently shown that the metabolic syndrome in pregnancy increases the risk for pre-eclampsia and GDM by two- to fourfold [27]. The metabolic syndrome in adults is associated with an approximately twofold higher risk for cardiovascular diseases and all-cause mortality [28]. There are clear links not only between cardiometabolic risk factors and accelerated ageing in adults [29] but also between maternal cardiovascular risk factors and cardiovascular risk factors in children [22-25]. Therefore, assessment of telomere length in children may serve as a marker of future development of cardiovascular and metabolic diseases.

The aims of this study are to determine whether presence of the metabolic syndrome in pregnant women associates with child telomere length or child anthropometry (weight, BMI) and BP, measured at 10 years of age.

\section{Methods}

Participants The Screening for Pregnancy Endpoints study (SCOPE) is a multicentre prospective cohort study that recruited nulliparous women with singleton pregnancies from Adelaide (Australia), Auckland (New Zealand), Cork (Ireland), Leeds, London and Manchester (UK) $(N=5628)$ between November 2004 and February 2011 [30]. Detailed data collection and inclusion criteria can be found in previous 
publications $[27,30]$. The current analysis consisted of women from the Adelaide SCOPE pregnancy cohort, who were recruited between November 2004 and September 2008. Maternal demographics, smoking status, family, medical and gynaecological history, anthropometry (height, weight, waist circumference) and BP were recorded at 14 16 weeks' gestation. Socioeconomic status was determined using the socioeconomic index (SEI), derived from the specific occupation of the woman, producing a score between 10 and 90 with a lower score reflecting greater disadvantage [31]. Ethnicity was binary coded as white/other. Smoking status was binary coded as yes/no for any cigarette smoking at the first visit. Ethical and practical constraints meant it was not appropriate to ask pregnant women to fast prior to their antenatal visit, thus a non-fasting blood sample was taken for measurement of HDL-cholesterol and triacylglycerol at 14 16 weeks' gestation. Plasma blood glucose was measured as a random blood sample by glucometer at the same time point. Details of immunoassay methodology for measuring lipids can be found in previous publications [32].

According to the IDF criteria for adults [33], a pregnant woman was considered to have the metabolic syndrome if she had a waist circumference $\geq 80 \mathrm{~cm}$ measured at the recruitment assessment (14-16 weeks' gestation), along with any two of the following: high triacylglycerol $(\geq 1.70 \mathrm{mmol} / 1$ $[\geq 150 \mathrm{mg} / \mathrm{dl}])$; low HDL-cholesterol $(<1.29 \mathrm{mmol} / 1$ $[<50 \mathrm{mg} / \mathrm{dl}]$ ); high BP (systolic BP $\geq 130 \mathrm{mmHg}$ or diastolic $\mathrm{BP} \geq 85 \mathrm{mmHg}$ ); or high fasting plasma glucose $(\geq 5.6 \mathrm{mmol} /$ 1). As this was a pregnancy cohort, a random plasma glucose sample was measured; however, the same concentration cutoff was used as in IDF criteria $(\geq 5.6 \mathrm{mmol} / \mathrm{l})$.

Information collected from the biological father included paternal age, and paternal height and weight (to calculate $\mathrm{BMI}$ ). Infant data (birthweight and sex) were recorded by research midwives within $72 \mathrm{~h}$ of birth.

Follow-up participants For follow-up, women from the SCOPE Adelaide cohort were contacted by telephone 810 years after their first pregnancy and were asked to attend a follow-up appointment with their child. Three attempts were made to contact each woman, using each of the telephone numbers specified in the medical records. If they did not respond, an SMS text was sent to any valid mobile telephone numbers informing them of the study and asking them to contact study coordinators. Of the 1164 women in the initial Adelaide cohort, we were able to contact 1139 (the remainder did not have valid phone numbers) (Fig. 1) and 270 mothers and their children attended the follow-up visit (24\% response rate). Included in the current analysis are 255 mother-child pairs for whom both a pregnancy blood sample (for assessment of the metabolic syndrome) and a follow-up child salivary DNA sample (for the primary outcome of telomere length) were available.

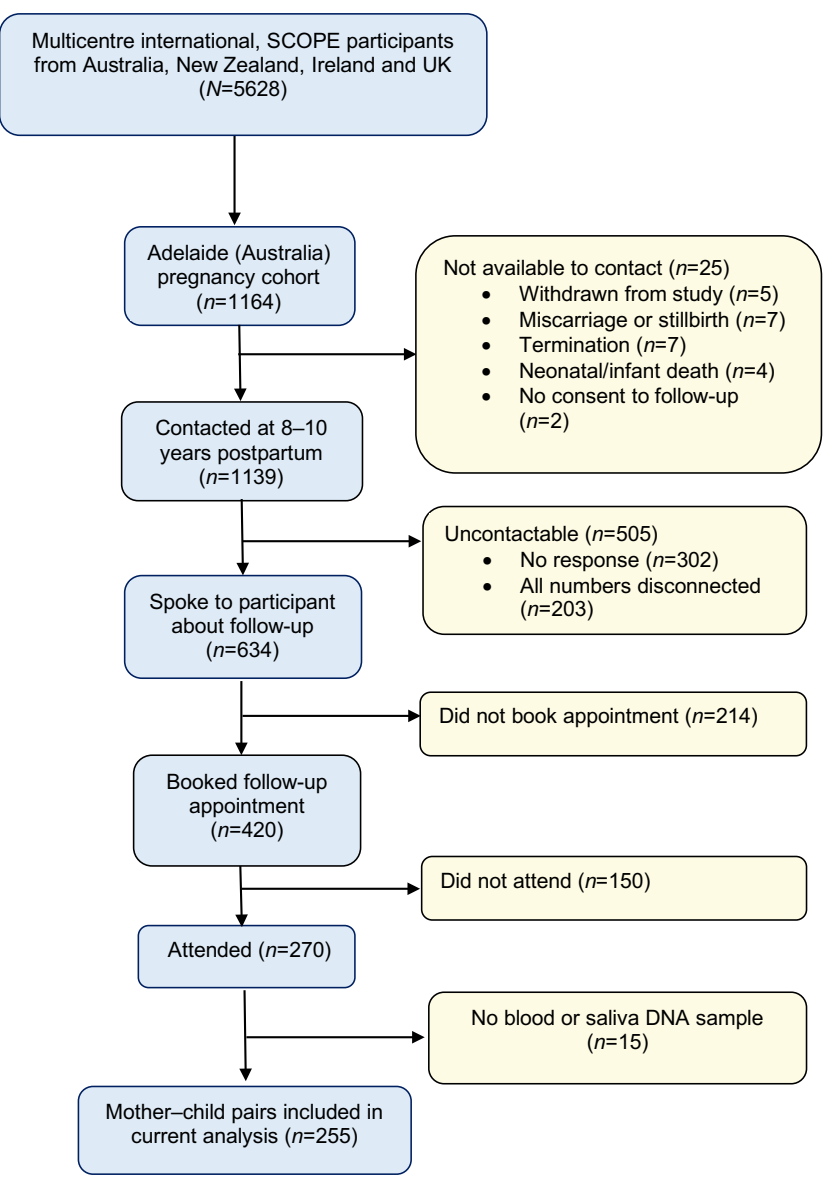

Fig. 1 Participant flow from initial SCOPE cohort through to SCOPE follow-up

Study visit During the follow-up visit, height, weight and BP were measured for the child. Height was measured with a stadiometer to the nearest $0.1 \mathrm{~cm}$; weight was measured to the nearest $0.1 \mathrm{~kg}$ using the TANITA SC-330 (Tokyo, Japan) bio-impedance scale, which also calculates BMI. Child peripheral systolic and diastolic BP was measured using an USCOM BP+ Sphygmomanometer (Brisbane, Australia). A saliva sample $(2 \mathrm{ml})$ was collected from the mother and her child, into an Oragene DNA OG-500 collection kit containing stabilisation solution (DNA Genotek, Canada). Saliva samples were stored at room temperature until DNA extraction was performed. At this visit, women and children provided written consent and assent, respectively. Ethics approvals for the SCOPE follow-up study were granted by the Human Research Ethics Committees of the University and hospitals [HREC/15/WCHN/192 and HREC/14/TQEH/277].

Outcome Child telomere length was the primary outcome. DNA was extracted from saliva samples using the prepIT L2P reagent (DNA Genotek) according to the manufacturer's instructions. DNA was allowed to gradually re-dissolve at $4{ }^{\circ} \mathrm{C}$ before DNA concentration was determined by NanoDrop One (Adelaide, Australia). DNA samples were diluted and 
transferred to 96 -well plates at a concentration of $2.5 \mathrm{ng} / \mu \mathrm{l}$. Ten-microlitre reactions were set up in each well of a 384-well white opaque PCR plate containing the following: $5 \mu$ of $2 \times$ SsoAdvanced Universal SYBR Green Supermix (Bio-Rad, USA); $100 \mathrm{nmol} / 1$ of each primer (either telomere (T) forward primer 5'-CGGTTTGTTTGGGTTTGGGTTTGGGT TTGGGTTTGGGTT-3' and telomere (T) reverse primer $5^{\prime}$ GGCTTGCCTTACCCTTACCCTTACCCTTACCCTT ACCCT-3' or 36B4 (S) forward primer 5'-CAGC AAGTGGGAAGGTGTAATCC-3' and 36B4 (S) reverse primer 5'-CCCATTCTATCATCAACGGGTACAA-3'); and $5 \mathrm{ng}$ of DNA [34]. Each PCR reaction was performed in triplicate. Pooled genomic DNA from unrelated samples was used as a control calibrator and was included on every plate. The quantitative PCR (qPCR) was run on a CFX384 Touch Real-Time PCR Detection System (Bio-Rad) with the following conditions: initial denaturation step of $3 \mathrm{~min}$ at $98^{\circ} \mathrm{C}$, followed by 40 cycles of $95^{\circ} \mathrm{C}$ for $5 \mathrm{~s}$ and $60^{\circ} \mathrm{C}$ for $30 \mathrm{~s}$. Melt curve analysis was performed at the end of 40 cycles of $65-95^{\circ} \mathrm{C}$, with $0.5^{\circ} \mathrm{C}$ increments at $2 \mathrm{~s}$ per step. Any samples that failed, or where the SD of the Cq value of triplicate wells was $>1$, were repeated or excluded from subsequent analyses. This generated a measure of the average telomere length as a ratio $(T / S)$ of telomere repeat length $(T)$ to copy number of a single-copy gene (S), for each DNA sample. The mean CV was $8.04 \%$ for the telomere PCR and $6.06 \%$ for genomic DNA PCR.

Maternal telomere length, measured from the saliva sample taken at follow-up, was included as a covariate in the statistical models.

Statistical analyses Frequencies and descriptive statistics for the characteristics of women and children are expressed as $n$ $(\%)$ or as mean $(95 \% \mathrm{CI})$. The impact of the metabolic syndrome on child telomere length was analysed in the following ways: (1) as a composite; (2) as each metabolic component; and (3) with an increasing number of components of the metabolic syndrome above the IDF cut-off point. These data were compared using a generalised linear model. A univariable model was first tested and then a multivariable model (model 1) adjusting for maternal age, SEI, ethnicity (white/other), smoking at first visit (yes/no), and maternal telomere length at follow-up, was run. A second multivariable model was then run, containing all factors from model 1 plus infant sex and birthweight. We then ran a third model, which further included paternal age and BMI. A supplementary analysis was investigated assessing the effect of the metabolic syndrome in the mother during pregnancy on child telomere length, by fetal sex. Causal diagrams (directed acyclic graphs) and content knowledge were used to guide selection of potential confounders. Children's BMI was converted to age- and sex-specific BMI $z$ scores (BMIz) using the WHO's Child Growth Standards, with height-for-age $z$ scores (HFAz) and weight-for-age $z$ scores (WFAz) calculated [35] using the WHO Anthro Survey Analyser R macro package [36]. The impact of the metabolic syndrome on child anthropometry (raw and $z$ scores) was then assessed using linear regression and the same models as described above. All analyses were conducted using SPSS (IBM SPSS Statistics for Windows, Version 24.0; IBM Corporation, Armonk, NY, USA).

\section{Results}

Participants Within the initial Adelaide SCOPE cohort, 22\% of women attended the 10 year follow-up visit with their child and are included in the current analysis. Mothers of children who did and did not participate (unable to contact or declined) in this study are compared in electronic supplementary material (ESM) Table 1. Compared with the women who did not attend the follow-up visit, women included in the study were older and had a higher SEI and BMI, and there was a higher percentage who were white and a lower percentage who smoked. The percentage of women who had the metabolic syndrome was similar in women who attended the follow-up and those who did not $(19.2 \%$ vs $16.0 \%)$. There were no differences in anthropometry of mothers who did and did not attend the follow-up (ESM Table 1).

Of the 255 women included in the current analysis, 20\% $(n=51)$ had the metabolic syndrome in pregnancy. Maternal and paternal characteristics at 14-16 weeks' gestation and child characteristics at follow-up are shown in Table 1. Of women who had the metabolic syndrome, all had high waist circumference, $92 \%$ had high glucose and $80 \%$ had high triacylglycerol. In women without the metabolic syndrome $(n=$ 204), $72 \%$ had a high waist circumference and $35 \%$ had high glucose. Women who had the metabolic syndrome in pregnancy had a higher mean BMI, larger waist circumference, higher systolic BP, higher random glucose, higher triacylglycerol and lower HDL-cholesterol (all $p<0.001$ ) compared with women without the metabolic syndrome. The mean (SD) age of children at follow-up was $9.6(0.6)$ years. There were no differences in child age, weight, BMI (raw or $z$ scores) or BP in children whose mothers did and did not have the metabolic syndrome in pregnancy (Table 1).

Association between the metabolic syndrome in a mother during pregnancy and child telomere length at $\mathbf{1 0}$ years of age The mean $(95 \% \mathrm{CI})$ child telomere length was 2.58 (2.43, 2.73). On univariable analysis, children of mothers who had the metabolic syndrome during pregnancy had shorter mean (95\% CI) telomere length than children of mothers without the metabolic syndrome $(2.27$ [1.94, 2.61] vs 2.65 [2.49, 2.82], $p=0.016$ ). After adjusting for maternal variables in model 1 , children of mothers who had the metabolic syndrome in pregnancy had $14 \%$ shorter telomeres than children of mothers 
Table 1 Maternal and paternal characteristics at 14-16 weeks' gestation and child characteristics at follow-up, comparing women who had vs did not have the metabolic syndrome in pregnancy

\begin{tabular}{|c|c|c|c|}
\hline Characteristic & No MetS $(n=204)$ & MetS $(n=51)$ & $p$ value \\
\hline \multicolumn{4}{|l|}{ Maternal characteristics } \\
\hline Age, years & $25.5(24.5,26.2)$ & $26.4(24.9,27.9)$ & 0.311 \\
\hline BMI, $\mathrm{kg} / \mathrm{m}^{2}$ & $27.2(26.3,28.1)$ & $31.4(29.3,33.5)$ & $<0.001$ \\
\hline White ethnicity, $n(\%)$ & $195(95.6)$ & $51(100)$ & 0.129 \\
\hline $\mathrm{SEI}^{\mathrm{a}}$ & $29.2(27.6,30.8)$ & $31.0(28.1,33.9)$ & 0.312 \\
\hline Smoking at first visit, no, $n(\%)$ & $175(85.8)$ & $51(100)$ & 0.226 \\
\hline \multicolumn{4}{|l|}{ Metabolic variables (continuous) } \\
\hline Waist circumference, $\mathrm{cm}$ & $88.8(86.9,90.7)$ & $99.1(94.9,103.4)$ & $<0.001$ \\
\hline $\mathrm{SBP}, \mathrm{mmHg}$ & $108.9(107.6,110.2)$ & $115.7(113.0,118.4)$ & $<0.001$ \\
\hline $\mathrm{DBP}, \mathrm{mmHg}$ & $64.3(63.2,65.3)$ & $66.6(64.2,69.0)$ & 0.065 \\
\hline Random glucose, mmol/1 & $5.45(5.36,5.54)$ & $6.19(5.94,6.43)$ & $<0.001$ \\
\hline Triacylglycerol, mmol/1 & $1.34(1.27,1.41)$ & $2.24(2.04,2.44)$ & $<0.001$ \\
\hline HDL-cholesterol, mmol/1 & $1.82(1.78,1.87)$ & $1.55(1.44,1.66)$ & $<0.001$ \\
\hline \multicolumn{4}{|l|}{ Metabolic variables (categorical), $n(\%)$} \\
\hline High waist circumference, $\geq 80 \mathrm{~cm}$ & $147(72)$ & $51(100)$ & $<0.001$ \\
\hline High SBP/DBP, $\geq 130 \mathrm{mmHg} \geq 85 \mathrm{mmHg}$ & $3(1.5)$ & $6(12)$ & 0.003 \\
\hline High random glucose, $\geq 5.6 \mathrm{mmol} / 1$ & $71(35)$ & $47(92)$ & $<0.001$ \\
\hline High triacylglycerol, $\geq 1.70 \mathrm{mmol} / 1$ & $30(15)$ & $41(80)$ & $<0.001$ \\
\hline Low HDL-cholesterol, <1.29 mmol/1 & $6(3)$ & $19(37)$ & $<0.001$ \\
\hline \multicolumn{4}{|l|}{ Paternal characteristics } \\
\hline Age, years & $28.3(27.4,29.3)$ & $30.0(28.3,31.8)$ & 0.868 \\
\hline BMI, $\mathrm{kg} / \mathrm{m}^{2}$ & $27.7(27.0,28.4)$ & $28.4(26.7,30.1)$ & 0.277 \\
\hline \multicolumn{4}{|l|}{ Follow-up data } \\
\hline Maternal telomere length & $2.23(2.06,2.39)$ & $2.08(1.81,2.35)$ & 0.368 \\
\hline \multicolumn{4}{|l|}{ Child characteristics } \\
\hline Male/female sex, $n(\%)$ & $91(46.6) / 113(55.4)$ & $21(41.2) / 30(58.8)$ & 0.390 \\
\hline Age, years & $9.63(9.55,9.70)$ & $9.55(9.38,9.72)$ & 0.321 \\
\hline Height, cm & $137.6(136.6,138.6)$ & $138.1(136.0,140.2)$ & 0.731 \\
\hline Height, $z$ score & $0.246(0.081,0.410)$ & $0.253(-0.025,0.531)$ & 0.369 \\
\hline Weight, kg & $34.4(33.1,35.8)$ & $36.1(33.3,38.8)$ & 0.797 \\
\hline Weight, $z$ score & $0.438(0.209,0.666)$ & $0.710(0.300,1.119)$ & 0.247 \\
\hline BMI, $\mathrm{kg} / \mathrm{m}^{2}$ & $17.9(17.4,18.4)$ & $18.7(17.6,19.9)$ & 0.401 \\
\hline BMI, $z$ score & $0.374(0.140,0.608)$ & $0.726(0.283,1.169)$ & 0.122 \\
\hline $\mathrm{SBP}, \mathrm{mmHg}$ & $111.4(109.6,113.1)$ & $110.2(107.2,113.2)$ & 0.171 \\
\hline DBP, mmHg & $60.0(58.7,61.3)$ & $59.7(57.7,61.7)$ & 0.319 \\
\hline
\end{tabular}

Data are presented as mean $(95 \% \mathrm{CI})$ or $n(\%)$

$p$ values were calculated using Student's $t$ test, test or $\chi^{2}$ test

${ }^{a} \mathrm{SEI}$ is a measure of socioeconomic status and is derived from the specific occupation of the woman, producing a score of 10-90, with a lower score reflecting greater disadvantage [31]

DBP; diastolic BP; MetS, the metabolic syndrome; SBP systolic BP without the metabolic syndrome (Table 2). When including fetal (model 2), and paternal factors (model 3), the estimates remained similar, with $14 \%$ shorter telomeres in children of mothers with the metabolic syndrome (model 3: mean difference -0.36 [95\% CI $-0.74,0.01])$.

Among the 255 included women, 32 (12.5\%) had no elevated components of the metabolic syndrome, 88 (34.5\%) had one elevated component, 83 (32.5\%) had two and 52 $(20.4 \%)$ had three or more. Table 3 shows the association between the metabolic syndrome in pregnancy (its individual components and increasing number of components) and child telomere length. Few women had a low waist circumference $(n=57)$, and most had high HDL-cholesterol $(n=230)$ or low BP $(n=246)$. Of the individual components of the metabolic 
Table 2 Associations between the metabolic syndrome in women during pregnancy and child telomere length ${ }^{\mathrm{a}, \mathrm{b}}$

\begin{tabular}{llll}
\hline & Model 1 $(n=255)$ & Model 2 $(n=255)$ & Model 3 $(n=235)$ \\
\hline Telomere length & $-0.37(-0.73,-0.02)$ & $-0.38(-0.74,0.26)$ & $-0.36(-0.74,0.01)$ \\
\hline
\end{tabular}

${ }^{a}$ Data are mean difference $(95 \% \mathrm{CI}$ ) representing presence vs absence of the metabolic syndrome in pregnancy

${ }^{\mathrm{b}}$ Telomere length is reported as a ratio $(\mathrm{T} / \mathrm{S})$ of telomere repeat length $(\mathrm{T})$ to copy number of a single-copy gene (S), therefore there are no units

Model 1 adjusted for maternal age, SEI, ethnicity (white/other), smoking at first visit (yes/no), maternal telomere length at follow-up; model 2 is model 1 plus infant sex and birthweight; model 3, is model 2 plus paternal age and BMI

syndrome, only waist circumference was associated with $12 \%$ shorter child telomeres, with similar mean $\mathrm{T} / \mathrm{S}$ ratios after adjusting for maternal factors in model 1 (low vs high waist circumference: 2.52 [2.08, 2.96] vs 2.94 [2.47, 3.41], $p=$ $0.019)$ and fetal and paternal factors $(2.51[2.06,2.29]$ vs 2.84 [2.35, 3.32], $p=0.082$ ). Compared with women who had no components of the metabolic syndrome, women who had $\geq 3$ of any components of the metabolic syndrome had children with 23\% shorter telomeres (adjusted mean [95\% CI] $1.82[1.01,2.62]$ vs $2.35[1.49,3.20], p=0.037$ ).

As shown in ESM Table 2, when stratified by sex, girls but not boys of mothers who had the metabolic syndrome in pregnancy had shorter telomere length than girls of mothers who did not have the metabolic syndrome in pregnancy (mean difference $-0.51[95 \% \mathrm{CI}-1.00,-0.01])$. However, there was no evidence of an interaction, such that the effect of the metabolic syndrome on child telomere length is not modified by sex, and is consistent for boys and girls (interaction term effect 0.18 [95\% CI $-0.57,0.93], p=0.6$ ).

\section{Metabolic syndrome in women during pregnancy and child} phenotype at 10 years of age In adjusted analyses, HFAz, WFAz and BMIz were similar in children from mothers who did and did not have the metabolic syndrome in pregnancy
(Table 4). Child systolic and diastolic blood pressures similarly did not differ between children of mothers who did or did not have the metabolic syndrome while pregnant.

\section{Discussion}

In this follow-up study of pregnant women and their 10-yearold children, the metabolic syndrome in early pregnancy was associated with $14 \%$ shorter telomeres in the child compared with children of mothers who did not have the metabolic syndrome. There were no differences in the children's anthropometry measures (raw or $z$ scores of weight-for-age, heightfor-age and BMI-for-age) or BP, when comparing offspring of mothers who did or did not have the metabolic syndrome.

Several studies have reported associations between intrauterine exposures and measurement of telomere length in placenta or cord blood leucocytes [37]. There is less information on maternal exposures in pregnancy and telomere length in children. A recent study $(n=439)$ demonstrated $14 \%$ shorter telomeres in 12-year-old children of mothers who had GDM, driven by shorter telomeres in female but not male children [38]. We demonstrated no effect modification with fetal sex, when assessing the metabolic syndrome and child telomere

Table 3 Association between individual components of the metabolic syndrome in women during pregnancy, and increasing number of components, and child telomere length ${ }^{\mathrm{a}}$

\begin{tabular}{|c|c|c|c|c|c|}
\hline Metabolic component & $n$ & Crude $(n=255)$ & Model $1(n=255)$ & Model $2(n=255)$ & Model $3(n=235)$ \\
\hline Low waist circumference, $<80 \mathrm{~cm}$ & 57 & $0.46(0.10,0.82)$ & $0.42(0.07,0.76)$ & $0.41(0.07,0.76)$ & $0.33(-0.04,0.69)$ \\
\hline High HDL-cholesterol, $\geq 1.29 \mathrm{mmol} / 1$ & 230 & $0.31(-0.19,0.82)$ & $0.31(-0.18,0.81)$ & $0.32(-0.17,0.81)$ & $0.30(-0.24,0.83)$ \\
\hline Low triacylglycerol, $<1.70 \mathrm{mmol} / \mathrm{l}$ & 184 & $0.17(-0.17,0.50)$ & $0.17(-0.15,0.49)$ & $0.17(-0.16,0.49)$ & $0.17(-0.17,0.51)$ \\
\hline Low glucose, $<5.6 \mathrm{mmol} / 1$ & 137 & $0.07(-0.24,0.37)$ & $0.05(-0.24,0.34)$ & $0.05(-0.24,0.34)$ & $0.04(-0.26,0.34)$ \\
\hline $\begin{array}{l}\text { Low systolic/diastolic BP, } \\
\quad<130 \mathrm{mmHg} /<85 \mathrm{mmHg}\end{array}$ & 246 & $0.27(-0.54,0.10)$ & $0.23(-0.55,1.00)$ & $0.25(-0.53,1.01)$ & $0.14(-0.64,0.92)$ \\
\hline \multicolumn{6}{|l|}{ No. of components } \\
\hline 0 & 32 & Reference & Reference & Reference & Reference \\
\hline 1 & 88 & $0.31(-0.17,0.79)$ & $0.22(-0.21,0.66)$ & $0.19(-0.24,0.63)$ & $0.25(-0.20,0.70)$ \\
\hline 2 & 83 & $0.25(-0.29,0.78)$ & $0.11(-0.39,0.62)$ & $0.11(-0.40,0.62)$ & $0.06(-0.47,0.58)$ \\
\hline$\geq 3$ & 52 & $0.61(0.13,1.09)$ & $0.54(0.08,1.09)$ & $0.55(0.07,1.02)$ & $0.53(0.03,1.03)$ \\
\hline
\end{tabular}

Data are mean difference $(95 \% \mathrm{CI})$ representing presence vs absence of the metabolic syndrome in pregnancy

Model 1 adjusted for maternal age, SEI, ethnicity (white/other), smoking at first visit (yes/no), maternal telomere length at follow-up; model 2 is model 1 plus infant sex and birthweight; model 3 is model 2 plus paternal age and BMI 
Table 4 Associations between the metabolic syndrome during pregnancy and child phenotype

\begin{tabular}{|c|c|c|c|c|c|c|c|c|c|}
\hline \multirow[t]{2}{*}{ Child characteristic } & \multicolumn{3}{|c|}{ Model 1} & \multicolumn{3}{|c|}{ Model 2} & \multicolumn{3}{|c|}{ Model 3} \\
\hline & $n$ & Mean difference $(95 \% \mathrm{CI})$ & $p$ value & $n$ & Mean difference $(95 \% \mathrm{CI})$ & $p$ value & $n$ & Mean difference $(95 \% \mathrm{CI})$ & $p$ value \\
\hline Height, cm & 252 & $-0.09(-2.33,2.16)$ & 0.940 & 252 & $-0.10(-2.12,-1.93)$ & 0.926 & 232 & $-0.12(-2.12,1.88)$ & 0.905 \\
\hline HFAz & 252 & $-0.11(-0.43,0.21)$ & 0.491 & 252 & $-0.12(-0.40,-0.17)$ & 0.434 & 232 & $-0.10(-0.39,0.19)$ & 0.491 \\
\hline Weight, kg & 191 & $-1.43(-4.38,1.52)$ & 0.342 & 191 & $-1.44(-4.19,1.32)$ & 0.307 & 191 & $-1.87(-4.47,0.74)$ & 0.160 \\
\hline WFAz ${ }^{\mathrm{a}}$ & 191 & $-0.28(-0.77,0.21)$ & 0.264 & 191 & $-0.28(-0.73,0.16)$ & 0.214 & 191 & $-0.19(-0.63,0.26)$ & 0.407 \\
\hline BMI, $\mathrm{kg} / \mathrm{m}^{2}$ & 252 & $-0.82(-1.95,0.31)$ & 0.155 & 252 & $-0.82(-1.90,0.27)$ & 0.139 & 232 & $-0.96(-2.00,0.08)$ & 0.071 \\
\hline $\mathrm{BMIz}^{\mathrm{a}}$ & 252 & $-0.34(-0.78,0.11)$ & 0.138 & 252 & $-0.34(-0.76,0.08)$ & 0.115 & 232 & $-0.37(-0.79,0.05)$ & 0.085 \\
\hline SBP, mmHg & 252 & $1.32(-2.45,5.08)$ & 0.493 & 252 & $1.33(-2.41,5.06)$ & 0.487 & 232 & $1.92(-1.80,5.63)$ & 0.312 \\
\hline $\mathrm{DBP}, \mathrm{mmHg}$ & 252 & $0.53(-2.21,3.26)$ & 0.705 & 252 & $0.52(-2.21,3.25)$ & 0.709 & 232 & $0.17(-2.35,2.70)$ & 0.893 \\
\hline
\end{tabular}

Model 1 adjusted for maternal age, SEI, ethnicity (white/other), smoking at first visit (yes/no), maternal telomere length at follow-up; model 2 is model 1 plus infant sex and birthweight; model 3, is model 2 plus paternal age and BMI

${ }^{\text {a }}$ BMIz is a height-based calculation (WHO child growth standards, 2017) and does not depend on the weight-for-age scores, which are only valid for children up to 10 years of age, hence smaller sample size with WFAz does not impact BMIz

DBP, diastolic BP; SBP, systolic BP

length, though our sample size was small. In a study of 1396 mother-child pairs, for each $1 \mathrm{~kg} / \mathrm{m}^{2}$ increase in maternal prepregnancy BMI, the 8-year-old child's telomere length was $0.23 \%$ shorter [39]. However, that study did not adjust for maternal telomere length or paternal age, both of which may influence offspring telomere length $[40,41]$. We have recently shown that women with the metabolic syndrome in early pregnancy had a near fourfold increased risk for GDM, independent of obesity [27]. The current study is unique in that the metabolic syndrome, a cluster of metabolic markers, was assessed at 14-16 weeks' gestation, far earlier than a diagnosis of GDM at 24-28 weeks' gestation. Identifying and potentially improving markers of the metabolic syndrome in early pregnancy may have a positive effect on telomere length in offspring, as well as their future disease risk.

Interestingly, when we assessed individual components of the metabolic syndrome, only high waist circumference $(>80 \mathrm{~cm})$ was associated with shorter child telomeres. This is somewhat surprising given that $78 \%$ of women had a high waist circumference, but supports the association described above with maternal pre-pregnancy BMI [39]. However, it is not so surprising that even shorter telomere lengths were seen when comparing children of women who had three or more markers of the metabolic syndrome with children of women who had no adverse metabolic components. While it has been shown that maternal metabolic markers change across gestation, and changes are more prominent in women who are obese or who have pre-existing hypertension of diabetes [42], it is unclear whether levels of metabolic components in our sample of low-risk women might be comparable to other study populations. As we are the first to report on individual and increasing number of components of the metabolic syndrome on child telomere length, further investigation into this area is warranted.
Mechanisms relating to the potential shortening of telomeres in children of mothers who had the metabolic syndrome in pregnancy are likely to be multifactorial. The metabolic syndrome encompasses a range of cardiovascular markers including raised lipids and $\mathrm{BP}$, insulin resistance and abdominal obesity, which are associated with increased oxidative stress and inflammation [43]. Chronic inflammation in adults leads to persistent damage to telomeres and increases the rate of telomere shortening, demonstrated in cross-sectional and longitudinal studies [4]. However, whether the association between the metabolic syndrome in women during pregnancy and shorter child telomeres observed in the present study is a consequence of adverse maternal intrauterine exposures, shared genetics or environmental factors occurring after birth is unknown. Additionally, even when adjusting for factors previously shown to be associated with telomere length, such as birthweight [44-46], the impact of the metabolic syndrome on child telomeres was still significant. Importantly, child telomere length has clinical relevance. Two longitudinal studies have shown that shorter telomeres measured in children at 3.6 years of age were associated with greater intima media thickness, a non-invasive measure of subclinical atherosclerosis, at both 8 years [47] and 14 years of age [48]. Early assessment of telomere length in children may therefore provide insight into their potential future disease risk.

A key finding of this study is that despite having shorter telomeres, there was no difference in anthropometry or BP in children of mothers who had the metabolic syndrome. This may suggest that biological/molecular ageing could occur before phenotypical changes are observed. We also found no association between child telomere length and child anthropometry at 10 years of age. Similar to our findings, Zannolli et al [49] found no difference in telomere length, measured in 
blood leucocytes, when comparing obese and normal-weight white children. In contrast, Al-Attas et al and Buxton et al reported shorter telomere length, also measured in blood leucocytes, in obese vs non-obese children aged 5-12 years [50] and 2-17 years [51]. Consistent with our findings, there was no association between BMI $z$ score and telomere length in the latter study [51]. Measurement of other characteristics of obesity such as elevated circulating lipids or inflammatory markers, which are observed in children who are obese [52], may assist in identifying childhood traits that are associated with biological/molecular ageing.

The study has several strengths. SCOPE was a populationbased prospective cohort design that included a large number of nulliparous women across six centres in four countries. Participants comprised a clearly defined population of nulliparous low-risk women with no pre-existing disease, utilising rigorous data collection techniques. The follow-up study captured 255 mother-child pairs for whom complete information on maternal metabolic variables and telomere length were available. Assessment of telomere length isolated from saliva DNA, a non-invasive source of DNA for biomarker assays, potentially allowed us to recruit larger numbers of a vulnerable population (i.e. children) than if a blood sample was required. The follow-up study also addressed limitations of previous studies that examined intrauterine exposures and child telomere length through inclusion of paternal and infant factors in the statistical models. Potentially, the greatest weakness of this study was that $23 \%$ of women in the initial Adelaide SCOPE cohort attended the 10 year follow-up with their respective child. It is unlikely that the reasons for dropout are related to the metabolic syndrome and telomere length, thus reasons for missing data cannot be sufficiently explained by the data we hold. To account for this in statistical analyses is difficult. From the initial 1139 women who consented to follow-up, we had a high rate of failure to contact these women (44\%) despite documentation of three different phone numbers, home address and maiden name information. Thirty four per cent of women were unwilling to cooperate; they were contacted but did not wish to participate $(n=214)$. While the rate of follow-up may appear low, compared with the Hyperglycemia and Adverse Pregnancy Outcome (HAPO) follow-up study in 10- to 14-yearold children, our rate of failure to locate participants in the current study $(44 \%)$ was similar to that study $(41 \%)$, we had a higher rate of women attending follow-up (64\% vs 52\%) and, as a proportion of eligible children, the rate of attendance was similar (23\% vs $26 \%$ ) [53]. Pending funding, we also plan to follow-up these children again at age 16 years when they are likely to have completed puberty. Regarding the telomere length assay, the qPCR CVs in the current study were $<11 \%$, indicating reproducibility within the assay. CVs can be influenced by a number of variables, including technical variation, biological variation and sample size. However, our study was performed within a single population, all DNA was collected, stored and extracted the same way, and by the same research team. Finally, although we cannot establish causation, our results provide supporting evidence on how maternal exposures might associate with offspring telomere length.

In conclusion, the metabolic syndrome in women during pregnancy is associated with shorter telomeres in their 8- to 10-year-old children, but not with differences in the children's anthropometry (BMI, $z$ scores of weight-for-age, height-forage) or BP. Further studies in larger cohorts of children are warranted, as well as investigation of whether telomere length measured in cord blood also associates with telomere length in childhood. Such studies would provide insight into the mechanism of telomere shortening and allow possible intervention strategies aimed at attenuating telomere shortening.

Acknowledgements The authors thank the SCOPE families who generously consented to participate in the follow-up study. We also thank D. McCullough for his assistance with laboratory work (Robinson Research Institute, University of Adelaide, Australia).

Data availability The SCOPE study, which commenced recruitment in 2004, did not seek specific consent from participants for sharing their data publicly. However, the SCOPE Consortium Scientific Advisory Board invites applications to use the collected data via e-mail to the chairperson, via A. Aherne at ei.ccu@enreha.yma. Applicants will be asked to complete a Research Application Form specifying details for their planned study which will then be reviewed by the SCOPE Scientific Advisory Board. The SCOPE Consortium is keen to promote collaboration among researchers and to see our unique SCOPE database and pregnancy biobank used in studies which meet our ethics and consenting process. The SCOPE Consortium is a member of the International Pregnancy Collaboration (https://pregnancycolab.tghn.org/) and has participated in several studies involving shared data. The SCOPE database is provided and maintained by MedSciNet AB (http:// medscinet.com).

Funding SCOPE was funded by Premier's Science and Research Fund, South Australian Government (http://www.dfeest.sa.gov.au/scienceresearch/premiers-research-and-industry-fund). The SCOPE follow-up study was funded by Channel 7 Children's Research Foundation Project Grant awarded to PHA, CTR and GAD (GNT 161305). JAG was supported by an Emerging Leadership Mentored Development Program, Faculty of Health and Medical Sciences, University of Adelaide and a Robinson Research Institute (RRI) Seed Grant (TBM, CTR, KLG, JAG). PHA was supported by an NHMRC Peter Doherty Early Career Fellowship (GNT1090778). CTR is supported by a Matthew Flinders Fellowship from Flinders University and a NHMRC Investigator Grant (GNT1174971). AG and MDP received PhD Research Training Program scholarships from the University of Adelaide.

Authors' relationships and activities The authors declare that there are no relationships or activities that might bias, or be perceived to bias, their work.

Contribution statement GAD, CTR and JAG made substantial contributions to the conception and design of the study. Substantial contribution was made by AG, MDP and PHA to acquisition of data, by DM, JAG and SYL to data analysis and by DM, JAG, SYL, LGS, TBM and KLG to interpretation of data. All authors were involved in drafting the article or revising it critically for important intellectual content. Final approval of the version to be published was given by all authors. JAG is the guarantor of this work. 


\section{References}

1. Blackburn EH, Epel ES, Lin J (2015) Human telomere biology: a contributory and interactive factor in aging, disease risks, and protection. Science 350(6265):1193-1198. https://doi.org/10. 1126/science.aab3389

2. Smith L, Luchini C, Demurtas J et al (2019) Telomere length and health outcomes: an umbrella review of systematic reviews and meta-analyses of observational studies. Ageing Res Rev 51:1-10. https://doi.org/10.1016/j.arr.2019.02.003

3. Houben JM, Moonen HJ, van Schooten FJ, Hageman GJ (2008) Telomere length assessment: biomarker of chronic oxidative stress? Free Radic Biol Med 44(3):235-246. https://doi.org/10.1016/j. freeradbiomed.2007.10.001

4. De Meyer T, Rietzschel ER, De Buyzere ML, Van Criekinge W, Bekaert S (2008) Studying telomeres in a longitudinal population based study. Front Biosci 13:2960-2970. https://doi.org/10.2741/ 2901

5. Adams JM, White M (2004) Biological ageing: a fundamental, biological link between socio-economic status and health? Eur J Pub Health 14(3):331-334. https://doi.org/10.1093/eurpub/14.3. 331

6. D'Mello MJ, Ross SA, Briel M, Anand SS, Gerstein H, Pare G (2015) Association between shortened leukocyte telomere length and cardiometabolic outcomes: systematic review and meta-analysis. Circ Cardiovasc Genet 8(1):82-90. https://doi.org/10.1161/ CIRCGENETICS.113.000485

7. Ma H, Zhou Z, Wei S et al (2011) Shortened telomere length is associated with increased risk of cancer: a meta-analysis. PLoS One 6(6):e20466. https://doi.org/10.1371/journal.pone.0020466

8. Cawthon RM, Smith KR, O'Brien E, Sivatchenko A, Kerber RA (2003) Association between telomere length in blood and mortality in people aged 60 years or older. Lancet 361(9355):393-395. https://doi.org/10.1016/S0140-6736(03)12384-7

9. Wang Q, Zhan Y, Pedersen NL, Fang F, Hagg S (2018) Telomere length and all-cause mortality: a meta-analysis. Ageing Res Rev 48: 11-20. https://doi.org/10.1016/j.arr.2018.09.002

10. Gorenjak V, Petrelis AM, Stathopoulou MG, Visvikis-Siest S (2020) Telomere length determinants in childhood. Clin Chem Lab Med 58(2):162-177. https://doi.org/10.1515/cclm-2019-0235

11. Liu B, Song L, Zhang L et al (2020) Prenatal second-hand smoke exposure and newborn telomere length. Pediatr Res 87(6):10811085. https://doi.org/10.1038/s41390-019-0594-2

12. Salihu HM, King LM, Nwoga C et al (2016) Association between maternal-perceived psychological stress and fetal telomere length. South Med J 109(12):767-772. https://doi.org/10.14423/SMJ. 0000000000000567

13. Salihu HM, Pradhan A, King L et al (2015) Impact of intrauterine tobacco exposure on fetal telomere length. Am J Obstet Gynecol 212(2):205 e201-205 e208. https://doi.org/10.1016/j.ajog.2014.08. 026

14. Martens DS, Cox B, Janssen BG et al (2017) Prenatal air pollution and newborns predisposition to accelerated biological aging. JAMA Pediatr 171(12):1160-1167. https://doi.org/10.1001/ jamapediatrics.2017.3024

15. Mirzakhani H, De Vivo I, Leeder JS et al (2017) Early pregnancy intrauterine fetal exposure to maternal smoking and impact on fetal telomere length. Eur J Obstet Gynecol Reprod Biol 218:27-32. https://doi.org/10.1016/j.ejogrb.2017.09.013

16. Martens DS, Plusquin M, Gyselaers W, De Vivo I, Nawrot TS (2016) Maternal pre-pregnancy body mass index and newborn telomere length. BMC Med 14(1):148. https://doi.org/10.1186/s12916016-0689-0
17. $\mathrm{Xu} \mathrm{J,} \mathrm{Ye} \mathrm{J,} \mathrm{Wu} \mathrm{Y} \mathrm{et} \mathrm{al} \mathrm{(2014)} \mathrm{Reduced} \mathrm{fetal} \mathrm{telomere} \mathrm{length} \mathrm{in}$ gestational diabetes. PLoS One 9(1):e86161. https://doi.org/10. 1371/journal.pone.0086161

18. Cross JA, Temple RC, Hughes JC et al (2010) Cord blood telomere length, telomerase activity and inflammatory markers in pregnancies in women with diabetes or gestational diabetes. Diabet Med 27(11):1264-1270. https://doi.org/10.1111/j.1464-5491.2010. 03099.x

19. Gilfillan C, Naidu P, Gunawan F, Hassan F, Tian P, Elwood N (2016) Leukocyte telomere length in the neonatal offspring of mothers with gestational and pre-gestational diabetes. PLoS One 11(10):e0163824. https://doi.org/10.1371/journal.pone.0163824

20. Blackmore HL, Ozanne SE (2013) Maternal diet-induced obesity and offspring cardiovascular health. J Dev Orig Health Dis 4(5): 338-347. https://doi.org/10.1017/S2040174412000761

21. Pathirana MM, Lassi ZS, Roberts CT, Andraweera PH (2020) Cardiovascular risk factors in offspring exposed to gestational diabetes mellitus in utero: systematic review and meta-analysis. $\mathrm{J}$ Dev Orig Health Dis 1-18. https://doi.org/10.1017/ S2040174419000850

22. Miliku K, Bergen NE, Bakker H et al (2016) Associations of maternal and paternal blood pressure patterns and hypertensive disorders during pregnancy with childhood blood pressure. J Am Heart Assoc 5(10):e003884. https://doi.org/10.1161/JAHA.116.003884

23. Gaillard R, Steegers EA, Duijts L et al (2014) Childhood cardiometabolic outcomes of maternal obesity during pregnancy: the Generation R Study. Hypertension 63(4):683-691. https://doi.org/ 10.1161/HYPERTENSIONAHA.113.02671

24. van Lieshout N, Oostvogels A, Gademan MGJ, Vrijkotte TGM (2017) Maternal early pregnancy lipid profile and offspring s lipids and glycaemic control at age 5-6 years: The ABCD study. Clin Nutr 36(6):1628-1634. https://doi.org/10.1016/j.clnu.2016.10.010

25. Dabelea D (2007) The predisposition to obesity and diabetes in offspring of diabetic mothers. Diabetes Care 30(Suppl 2):S169S174. https://doi.org/10.2337/dc07-s211

26. Huang PL (2009) A comprehensive definition for metabolic syndrome. Dis Model Mech 2(5-6):231-237. https://doi.org/10. 1242/dmm.001180

27. Grieger JA, Bianco-Miotto T, Grzeskowiak LE et al (2018) Metabolic syndrome in pregnancy and risk for adverse pregnancy outcomes: a prospective cohort of nulliparous women. PLoS Med 15(12):e1002710. https://doi.org/10.1371/journal.pmed.1002710

28. Mottillo S, Filion KB, Genest J et al (2010) The metabolic syndrome and cardiovascular risk a systematic review and metaanalysis. J Am Coll Cardiol 56(14):1113-1132. https://doi.org/10. 1016/j.jacc.2010.05.034

29. Smeets CC, Codd V, Samani NJ, Hokken-Koelega AC (2015) Leukocyte telomere length in young adults born preterm: support for accelerated biological ageing. PLoS One 10(11):e0143951. https://doi.org/10.1371/journal.pone.0143951

30. North RA, McCowan LM, Dekker GA et al (2011) Clinical risk prediction for pre-eclampsia in nulliparous women: development of model in international prospective cohort. BMJ 342:d1875. https:// doi.org/10.1136/bmj.d1875

31. Davis P, McLeod K, Ransom M, Ongley P (1997) The New Zealand socio-economic index of occupational status (NZSEI) (Research report no. 2). Wellington: Statistics NZ. 1997. Available from http://www2.stats.govt.nz/domino/external/web/ prod_serv.nsf/874ea91c142289384c2567a80081308e/ dc0ac1758236cb28cc256cef00748da5/\$FILE/NZSEI.pdf. Accessed 16 July 2019

32. Fyfe EM, Rivers KS, Thompson JM et al (2013) Elevated maternal lipids in early pregnancy are not associated with risk of intrapartum caesarean in overweight and obese nulliparous women. BMC Pregnancy Childbirth 13:143. https://doi.org/10.1186/1471-239313-143 
33. International Diabetes Federation (2006) IDF Consensus Worldwide Definition of the Metabolic Syndrome. The IDF consensus worldwide definition of the metabolic syndrome. Available from file://C:/Users/a1068108/Downloads/ IDF_Meta def final.pdf. Accessed 12 June 2019

34. O'Callaghan NJ, Fenech M (2011) A quantitative PCR method for measuring absolute telomere length. Biol Proced Online 13:3. https://doi.org/10.1186/1480-9222-13-3

35. World Health Organization (2006) WHO child growth standards. Length/height-for-age, weight-for-age, weight-for-length, weightfor-height and body mass index-for-age. Methods and development. Available from https:/www.who.int/childgrowth/standards/ Technical_report.pdf. Accessed 16 July 2019

36. World Health Organization (2010) Child growth standards. WHO anthro survey analyser and other tools. Available from https://www. who.int/childgrowth/software/en/. Accessed 16 July 2019

37. Entringer S, de Punder K, Buss C, Wadhwa PD (2018) The fetal programming of telomere biology hypothesis: an update. Philos Trans R Soc Lond Ser B Biol Sci 373(1741):20170151. https:// doi.org/10.1098/rstb.2017.0151

38. Hjort L, Vryer R, Grunnet LG et al (2018) Telomere length is reduced in 9- to 16-year-old girls exposed to gestational diabetes in utero. Diabetologia 61(4):870-880. https://doi.org/10.1007/ s00125-018-4549-7

39. Clemente DBP, Maitre L, Bustamante M et al (2019) Obesity is associated with shorter telomeres in 8 year-old children. Sci Rep 9(1):18739. https://doi.org/10.1038/s41598-019-55283-8

40. Nguyen MT, Lycett K, Vryer R et al (2019) Telomere length: population epidemiology and concordance in Australian children aged 11-12 years and their parents. BMJ Open 9(Suppl 3):118-126. https://doi.org/10.1136/bmjopen-2017-020263

41. Kimura M, Cherkas LF, Kato BS et al (2008) Offspring s leukocyte telomere length, paternal age, and telomere elongation in sperm. PLoS Genet 4(2):e37. https://doi.org/10.1371/journal.pgen. 0040037

42. Oostvogels A, Busschers WB, Spierings EJM, Roseboom TJ, Gademan MGJ, Vrijkotte TGM (2017) Pre-pregnancy weight status, early pregnancy lipid profile and blood pressure course during pregnancy: the ABCD study. PLoS One 12(5):e0177554. https://doi.org/10.1371/journal.pone.0177554

43. Carrier A (2017) Metabolic syndrome and oxidative stress: a complex relationship. Antioxid Redox Signal 26(9):429-431. https://doi.org/10.1089/ars.2016.6929
44. de Zegher F, Diaz M, Lopez-Bermejo A, Ibanez L (2017) Recognition of a sequence: more growth before birth, longer telomeres at birth, more lean mass after birth. Pediatr Obes 12(4):274 279. https://doi.org/10.1111/ijpo.12137

45. Lee SP, Hande P, Yeo GS, Tan EC (2017) Correlation of cord blood telomere length with birth weight. BMC Res Notes 10(1): 469. https://doi.org/10.1186/s13104-017-2791-6

46. Wojcicki JM, Olveda R, Heyman MB et al (2016) Cord blood telomere length in Latino infants: relation with maternal education and infant sex. J Perinatol 36(3):235-241. https://doi.org/10.1038/ jp. 2015.178

47. Skilton MR, Nakhla S, Ayer JG et al (2016) Telomere length in early childhood: Early life risk factors and association with carotid intima-media thickness in later childhood. Eur J Prev Cardiol 23(10):1086-1092. https://doi.org/10.1177/2047487315607075

48. Barraclough JY, Skilton MR, Garden FL, Toelle BG, Marks GB, Celermajer DS (2019) Early and late childhood telomere length predict subclinical atherosclerosis at age 14yrs. - the CardioCAPS study. Int J Cardiol 278:250-253. https://doi.org/10.1016/j.ijcard. 2018.12.065

49. Zannolli R, Mohn A, Buoni S et al (2008) Telomere length and obesity. Acta Paediatr 97(7):952-954. https://doi.org/10.1111/j. 1651-2227.2008.00783.x

50. Al-Attas OS, Al-Daghri N, Bamakhramah A, Shaun Sabico S, McTernan P, Huang TT (2010) Telomere length in relation to insulin resistance, inflammation and obesity among Arab youth. Acta Paediatr 99(6):896-899. https://doi.org/10.1111/j.1651-2227.2010. 01720.x

51. Buxton JL, Walters RG, Visvikis-Siest S, Meyre D, Froguel P, Blakemore AI (2011) Childhood obesity is associated with shorter leukocyte telomere length. J Clin Endocrinol Metab 96(5):1500 1505. https://doi.org/10.1210/jc.2010-2924

52. Higgins V, Omidi A, Tahmasebi H et al (2020) Marked influence of adiposity on laboratory biomarkers in a healthy cohort of children and adolescents. J Clin Endocrinol Metab 105(4):e1781-e1797. https://doi.org/10.1210/clinem/dgz161

53. Scholtens DM, Kuang A, Lowe LP et al (2019) Hyperglycemia and Adverse Pregnancy Outcome Follow-up Study (HAPO FUS): maternal glycemia and childhood glucose metabolism. Diabetes Care 42(3):381-392. https://doi.org/10.2337/dc18-2021

Publisher's note Springer Nature remains neutral with regard to jurisdictional claims in published maps and institutional affiliations. 